\title{
2.23 AND 8.29 GHZ OBSERVATIONS OF CSS-GPS CANDIDATES
}

\author{
D. DALLACASA, M. BONDI, F. MANTOVANI
}

Istituto di Radioastronomia - CNR, Bologna, Italy

AND

W. ALEF

Max Planck Institut für Radioastronomie, Bonn

Compact Steep-Spectrum (CSS) and GHz-Peaked Spectrum (GPS) radio sources are intrinsically small objects (Fanti et al. 1990) with high frequency steep spectra $\left(\alpha>0.5\right.$ with $\left.S \propto \nu^{-\alpha}\right)$, found at moderate or high redshifts $(z>0.2$ but many objects have $z>1.5)$. Their characteristics have been interpreted in terms of youth (e.g. Fanti et al. 1995) or "frustration" (e.g. van Breugel et al. 1984). Their radio spectra turn over at tens or a few hundreds of $\mathrm{MHz}$ (CSS) or at higher frequencies (GPS), interpreted as due to synchrotron self-absorption. They represent a significant fraction of flux limited catalogues (15-30\%, depending on the frequency).

The samples studied so far (Spencer et al. 1989, Fanti et al. 1990, Dallacasa et al. 1995) were limited to the most powerful objects. Sources with lower power will help in understanding the relation between the CSS-GPS objects and other classes of radio sources.

We present the results of $\mathrm{S} / \mathrm{X}(2.23 / 8.29 \mathrm{GHz})$ VLBI snapshot observations of 29 sources drawn from a list of CSS and GPS candidates. Data were recorded in Mk3 mode A during two separate VLBI sessions using the antennas in Effelsberg, Medicina, Noto, Onsala, Matera and Wettzell. Typical resolutions were about $30 \times 12$ mas and $9 \times 3.5$ mas at $S$ and $X$ bands respectively. Each source was observed for a number (3-5) of 13-min scans in a range of hour angle and due to the limited uv-coverage only simple structures could be imaged. Only two sources were not detected. The r.m.s. noise level in the images is typically of $1-2 \mathrm{mJy}$ and the dynamic range achieved is between a few tens and a few hundreds. A summary of the parameters from the images is presented in Table 1. 
TABLE 1. Summary of the observational results: In column $3, P=$ pointlike; $S=$ single component, resolved either at $\mathrm{S}$ or at $\mathrm{X}$ band (or both); $\mathrm{D}=$ double; $\mathrm{C}-\mathrm{J}=$ core-jet; $\mathrm{T}$ $=$ triple; $C P L X=$ complex. $N D=$ Not Detected. The flux densities reported in column $4\left(S_{C F D}\right)$ and column $6\left(X_{C F D}\right)$ refer to the integrated values measured on the images.

\begin{tabular}{|c|c|c|c|c|c|c|}
\hline IAU Name & $\begin{array}{l}\text { LAS } \\
\text { mas }\end{array}$ & Morph. & $\begin{array}{c}\mathbf{S}_{C F D} \\
\mathrm{Jy}\end{array}$ & $\begin{array}{c}\mathrm{HPBW}_{S} \\
\operatorname{mas}(\mathrm{p} . \mathrm{a})\end{array}$ & $\begin{array}{c}\mathrm{X}_{C F D} \\
\mathbf{J y}\end{array}$ & $\begin{array}{l}\mathrm{HPBW}_{X} \\
\operatorname{mas}(\mathbf{p} . \mathrm{a})\end{array}$ \\
\hline $0201+113$ & & $\mathbf{P}$ & 0.56 & $25.1 \times 10.5(60)$ & 0.67 & $9.7 \times 3.3(53)$ \\
\hline $0237-027$ & & $\mathbf{S}$ & 0.26 & $20.9 \times 13.7(58)$ & 0.80 & $7.5 \times 4.3(64)$ \\
\hline $0237-233$ & & S? & 3.27 & $49.7 \times 16.9(37)$ & 1.93 & $21.0 \times 4.5(33)$ \\
\hline $0500+019$ & 10 & $\mathbf{S}$ & 1.56 & $26.2 \times 11.4(70)$ & 1.36 & $9.8 \times 3.4(68)$ \\
\hline $0511-220$ & & S?-CJ? & 0.33 & $38.2 \times 19.2(42)$ & 0.86 & $14.7 \times 5.4(49)$ \\
\hline $0743-006$ & & $\mathbf{P}$ & 0.70 & $26.7 \times 13.0(-78)$ & 1.71 & $8.4 \times 4.5(84)$ \\
\hline $0922+005$ & & S?-CJ? & 0.45 & $24.4 \times 12.5(-73)$ & 0.41 & $10.0 \times 4.5(-87)$ \\
\hline $0941-080$ & 50 & D & 0.92 & $37.9 \times 18.0(-57)$ & 0.20 & $8.2 \times 5.4(-81)$ \\
\hline $0941+261$ & - & - & ND & - & ND & - \\
\hline $1143-245$ & & $\mathbf{S}$ & 1.11 & $40.7 \times 20.3(41)$ & 0.92 & $16.8 \times 6.0(39)$ \\
\hline $1237-101$ & & $\mathbf{S}$ & 0.82 & $23.4 \times 14.3(51)$ & 0.67 & $7.9 \times 4.2(47)$ \\
\hline $1317-005$ & & $\mathbf{P}$ & 0.18 & $20.9 \times 13.0(-54)$ & 0.11 & $7.1 \times 3.8(50)$ \\
\hline $1402-012$ & & $\mathbf{P}$ & 0.49 & $19.1 \times 11.8(-83)$ & 0.29 & $5.2 \times 3.3(-84)$ \\
\hline $1502+036$ & & $\mathbf{S}$ & 0.51 & $28.6 \times 11.3(56)$ & 0.61 & $13.5 \times 3.2(46)$ \\
\hline $1518+047$ & 150 & D & 1.69 & $22.1 \times 10.2(53)$ & 0.40 & $10.5 \times 3.6$ \\
\hline $1602+576$ & 120 & D? & 0.57 & $17.8 \times 10.6(89)$ & 0.34 & $4.1 \times 2.4(90)$ \\
\hline $1607+268$ & 60 & D & 2.39 & $18.7 \times 9.9(66)$ & 0.90 & $9.1 \times 3.7(48)$ \\
\hline $1629+120$ & 1150 & D & 0.29 & $31.9 \times 10.0(53)$ & 0.11 & $10.0 \times 4.1(44)$ \\
\hline $1629+680$ & 120 & C-J & 0.79 & $20.6 \times 11.0(84)$ & 0.20 & $5.2 \times 3.5(70)$ \\
\hline $1801+010$ & & $\mathbf{P}$ & 1.64 & $21.6 \times 13.6(68)$ & 1.05 & $5.5 \times 3.2(80)$ \\
\hline $1848+283$ & & $\mathbf{P}$ & 0.24 & $22.1 \times 10.6(54)$ & 1.44 & $8.3 \times 3.4(51)$ \\
\hline 2044-027 & 40 & $\mathbf{S}$ & 0.29 & $28.0 \times 17.9(62)$ & 0.03 & $10.0 \times 4.3(50)$ \\
\hline 2053-201 & - & - & ND & $\longrightarrow$ & ND & $\longrightarrow$ \\
\hline 2126-158 & & $\mathbf{P}$ & 0.56 & $33.6 \times 14.7(42)$ & 0.86 & $13.0 \times 3.9(41)$ \\
\hline $2128+048$ & 60 & $\mathrm{D}$ ? & 2.25 & $24.1 \times 12.1(61)$ & 1.28 & $8.1 \times 3.7(53)$ \\
\hline $2137+209$ & 50 & $\mathbf{S}$ & 0.63 & $24.6 \times 9.8(52)$ & 0.23 & $9.6 \times 3.3(46)$ \\
\hline $2210+016$ & 100 & T-CPLX & 1.20 & $23.6 \times 12.3(60)$ & 0.40 & $9.7 \times 3.7(54)$ \\
\hline $2223+210$ & & $\mathbf{S}$ & 0.79 & $22.3 \times 10.0(57)$ & 1.23 & $8.8 \times 2.9(50)$ \\
\hline 2351-006 & & $\mathbf{S}$ & 0.29 & $24.2 \times 12.6(60)$ & 0.27 & $8.7 \times 3.9(65)$ \\
\hline
\end{tabular}

\section{References}

van Breugel, W.J.M., Miley, G.K., Heckman, T.M. 1984, AJ, 89, 5.

Dallacasa, D., et al. 1995, $A \& A, 295,27$.

Fanti, C., et al. 1995, $A \& A, 302,317$.

Fanti, R., et al. 1990, $A \& A, 281,333$.

Spencer, R.E., et al. 1989, MNRAS, 240, 657. 